\title{
Optimization process of moringa oleifera seed extract using artificial neural network (ANN)
}

\author{
Munirat Abolure Idris a, ${ }^{\text {, }}$, Mohammed Saedi Jami ${ }^{b}$, Ademola Monsur Hammed ${ }^{a}$ \\ ${ }^{a}$ International Institute for Halal Research and Training, International Islamic University, Malaysia \\ ${ }^{b}$ Department of Biotechnology Engineering, International Islamic University, Malaysia \\ * Corresponding author: munirat@iium.edu.my
}

\section{Article history}

Received 27 February 2018

Revised 28 Mac 2018

Accepted 21 May 2018

Published Online 14 April 2019

\begin{abstract}
The use of chlorine which causes disinfection of by-products is a major concern especially in the developed countries. There is the need to look for a cheap alternative such as the use of plant materia as substitute for chemical disinfectant. Moringa oleifera is an extensively documented plant material used for the treatment of drinking water. Its seed extracts contain active agents that having excellent coagulation properties and exerting in-vitro bactericidal activity. However, lack of available literature on the statistical optimization using artificial neural network (ANN) for inactivation kinetics of the seed extract using different disinfection models is the major aspect that need to be explored. This study was conducted to develop operation parameters using ANN for the seed extracts to be used as disinfectant for water treatment. The optimization process based on statistical experimental design using artificial neural network (ANN) in MATLAB 2012A was used to identify and determine the optimum process conditions. The multivariate regression analysis of the disinfection kinetic models was analyzed using SPSS version 20 and the final application of the optimized process conditions with river water was evaluated. The statistical analysis of optimization results using ANN gave a high coefficient of determination (R2) of 0.9992 and 0.9886 for E.coli. The model developed was verified and the optimum process parameters were $124 \mathrm{mg} / \mathrm{L}$ dosage, 65 minutes of contact time, $110 \mathrm{rpm}$ mixing rate for E.coli bacterial strain. The order of reaction followed second order and the inactivation kinetics showed that modified Hom model was best fitted the disinfection process with R2 of 0.711 . The findings from the application of the seed extract to river water showed that the removal efficiency for the seed extract with over $99.98 \%$ reduction of heterotrophic bacteria after the disinfection process. Hence, the findings of this study showed that defatted Moringa oleifera seed extract using the salt extraction method could be used as a disinfectant. This extract was recommended to be used in small communities and in emergency situations.
\end{abstract}

Keywords: Moringa oleifera seed, artificial neural network, E.coli, heterotrophic bacteria

\section{INTRODUCTION}

Artificial neural network (ANN) is a computational model that functions like a human brain through biological neurons and it is being applied as an alternative to determine classification, pattern completion and recognition, time series modelling, optimization etc (Haykin, 1999). It has the ability to manage data from non-lineal, adaptive and parallel process (Alvarez, 2006) and it has diverse useful applications, particularly in the area of image processing, remote sensing, approximation of function, prediction of behaviour of an event that changes with time, and optimization processes. The determination of the optimum values of properties such as the learning function, activation and transfer functions is the major issue that will be encountered due to the absence particular theories and principles that explaining the use of data set. Hence, using trial and error procedures is assumed to be the best tool for the optimization of neural network topology. The Feed-forward neural network is a commonly used neural network where the connections between input, hidden and output nodes are only connected in forward direction with no backward loops entirely from the structure. The feed forward neural network uses the back-propagation (BP) algorithm which involves the training of multilayer feed-forward neural network that comprising of non-linear units. It has a simple gradient descent method that designed to lower the total error or mean error of the output computed by the network. When using the BP algorithm, the errors for the input data are propagated backwards, from the output layer towards the input layer, with the corrections to the weights selected to minimize the residual error between actual and desired outputs. The topography of multi-layer feed-forward network comprises of an input layer, output layer and one or more layers between the input and the output. The Feed-forward back-propagation learns through a supervised process with pre-set inputs and target sets of data obtained from an existing system. During the learning process, two steps are involved; the inputs are propagated forward through the network and the error between the network outputs 
and targets are computed and propagated backward. This is the popular objective of determining the network weights through backpropagation algorithm involving trade-off between the cost and performance function (Wu et al., 2011). Generally, the backpropagation algorithm is a function of Least Means Square (LMS) method, using gradient search to minimize the cost function and the Mean Square Error (MSE) between the target and the actual network output (Lippmann, 1987) and the most commonly used cost function is given by;

$$
E=\sum_{j}^{k}\left\{y_{k}(x ; \boldsymbol{w})-t\right\}^{2}
$$

where $E$ in the equation 1 represents the MSE which is the function of the weight vector $\boldsymbol{w}$, and can be minimized when appropriate values of $\boldsymbol{w}$ are chosen. Iterative technique is required to minimize the error function $E$ by selecting small random values of $\boldsymbol{w}$ for the beginning of the training. The weight update algorithm is represented as;

$$
w_{k+1}=w_{k}+\lambda_{k} d_{k}
$$

where $\boldsymbol{w}_{k}$ is the current weights and biases vector, $\boldsymbol{d}_{k}$ is the current gradient and $\lambda_{k}$ is the learning rate. The update of the weights and biases vectors can be done either batch wise or incremental wise. In batch mode training, the weights and biases are updated only after all the entire training sets have been applied to the network, while in the incremental mode, the weights and biases are updated after each input is applied to the network.

The direction of the gradient descent vector $\boldsymbol{d}_{k}$ in equation 2 determines the convergence rate and computational complexity. The task of activation function is to limit the neuron response between the asymptotic values and it is represented by a symbol $f$ in equation 3 . There are various activation functions used in the training of neural network that includes linear, step, sigmoid (log-sigmoid) and hyperbolic-tan (tan-sigmoid). The linear transfer function is mostly used in the output layer of multilayer Perceptrons. The linear transfer function returns the exact value that passed through it as the output and it can be binary (Equation 3) or bipolar (Equation 4) and the two conditions are expressed as:

$$
\begin{aligned}
& f\left(\sum_{i=0}^{p} w_{k i} x_{i}+b\right)=\left\{\begin{array}{l}
1, \quad \text { if } \sum_{i=0}^{p} w_{k i} x_{i}+b \geq 0 \\
0, \text { if } \quad \sum_{i=0}^{p} w_{k i} x_{i}+b<0
\end{array}\right. \\
& f\left(\sum_{i=0}^{p} w_{k i} x_{i}+b \Theta\right)=\left\{\begin{array}{cc}
1 & \text { if } \sum_{i=0}^{p} w_{k i} x_{i}+b \geq 0 \\
-1, \text { if } & \sum_{i=0}^{p} w_{k i} x_{i}+b<0
\end{array}\right.
\end{aligned}
$$

The sigmoid transfer function looks like S-shape graphically and it is the most widely activation function used in artificial neural network applications (Haykin, 1999). There are various types of sigmoid functions but the most commonly used are log-sigmoid and the functions of the log-sigmoid and hyperbolic tangent, which are expressed in Equation 5 and 6, respectively.

$$
\begin{array}{r}
f\left(\sum_{i=0}^{p} w_{k i} x_{i}+b \theta\right)=\frac{1}{1+e^{-\lambda\left(a_{k}\right)}} \\
f\left(\sum_{i=0}^{p} w_{k i} x_{i}+b \theta\right)=\frac{e^{\lambda\left(a_{k}\right)}-e^{\lambda\left(a_{k}\right)}}{e^{\lambda\left(a_{k}\right)}+e^{\lambda\left(a_{k}\right)}}
\end{array}
$$

where $a_{k}$ (same parameter in equation 6) is the slope parameter that controls the steepest of the function, and $a_{k}$ is $\left(\sum_{i=0}^{p} w_{k i} x_{i}+b \theta\right)$. The output range of log-sigmoid is binary ( 1 or 0$)$ while that of hyperbolic tangent is bipolar $(+1$ or -1$)$. The activation function in the output layer is generally linear in almost any type of neural network applications. But the activation function in the hidden layer may be Log-sigmoid or Hyperbole tangent that depends on the layers responses. Though there is no particular theory in which to select the activation function in the hidden layer, a number of design parameters can affect the performance of ANN. These parameters include the choice of transfer function, training algorithm, and training parameters such as learning rate and momentum, number of hidden layers, number of hidden neurons in each hidden layer, initial weights, and training duration. There is no easy way to determine the best parameters of ANN model architecture without using all possible values of parameters and estimating the error of each (Mahajan et al., 2006). All created ANN models are feedforward backpropagation type of network. The best models after training are selected according to the multiple linear regression coefficient (R) and mean squared error (MSE) values. The closer the value of $R$ to 1 , the better the model is. While, the $R$ that close to 0 indicates that the model is still poor and need to be improved (Demuth, 2008). In addition, the best trained models are only considered into analysis when the best validation performance after training is not at epoch 0 .

The sensitivity analysis is done by using the linear correlation in MATLAB, which works by linear correlation that quantifies the strength of a linear relationship between two variables. When there is no correlative relationship between the two values, then the values of one quantity to increase or decrease with the values of the second quantity is nonexistence. The correlation coefficients range from - 1 to 1 , where values are close to 1 that suggesting a positive linear relationship between the data columns. When values are close to -1 that suggesting a negative linear relationship between data. The values that are close to or equal to 0 that suggesting no linear relationship.

In this study, the properties of the neural networks such as training or/learning function, activation function, numbers of nodes in the hidden layer would be investigated using the data obtained from the total viable count of E.coli cells in order to obtain an optimum NN topology for this study. This work therefore, was intended to focus on the use of other mathematical techniques such as ANN to carry out the optimization study of the process parameters.

\section{LITERATURE REVIEW}

Artificial Neural Network (ANN) draws its strength from management of non-lineal, adaptive and parallel process (Alvarez, 2006). It has diverse useful and successful applications particularly in the area of image processing, remote sensing, approximation of function, prediction of behaviour of an event that changes with time, and optimization which determines solution that decreases or increases an objective function.

There are lots of researchs done on the use of ANN particularly in the optimization of various bioprocess applications such as lipase production (Ricca et al., 2012), bio-surfactant production ( $\mathrm{Pal}$ et al., 2009) water and wastewater treatment. Research on the use of Moringa oleifera seed extract is focused on its coagulation ability for the treatment of water, as a result, the bacteriology assessment is only done to quantify the remaining bacteria left in water. Hence, there has been very little research on the use of the seed extract as disinfectant although few researchers (Bichi et al., 2012) have done the optimization study on the process parameters of the seed extract as a disinfectant using Face Centered Central Composite Design (FCCCD) under the response surface methodology (RSM). It is noteworthy to mention that FCCCD is the most commonly used technique for optimization, hence there is need to explore other optimization techniques such as artificial intelligence. It is pertinent to note that no optimization study has been done by using ANN for moringa oleifera used as disinfectant, hence this study would attempt to use ANN for the optimization process. Similarly, other researchers (Nwaiwu \& Lingmu, 2011) and (Bichi et al., 2012) have also explored the disinfection kinetics of the seed extract. In their work, only the first order models (Chicks law and Watson law) were explored to explain the disinfection inactivation mechanisms.

\section{METHODOLOGY/MATERIALS}

\section{Raw material}

The main material used in this study was dry Moringa oleifera seed which was collected from villages surrounding Bayero University (New Campus), Kano, Nigeria. 


\section{Water sample}

Raw water samples were collected from Sungai Gombak (a drinking source used by Puncak Niaga). The water sample was characterized and the values obtained from the characterization were used to prepare the synthetic water that was used throughout the study.

\section{Extracts preparation}

Dried Moringa oleifera seeds were de-husked and ground using a blender into fine powder. This powder was sieved through $210 \mu \mathrm{m}$ sieve to collect very fine powder for the oil extraction process.

\section{Bacterial strains}

Bacteria strains (E.coli) were locally obtained from available stocks presented in the Microbiology laboratory of Biotechnology Department, International Islamic University Malaysia and maintained on LB (Merck Sdn Bhd) slants. The strains were sub-cultured every two weeks and stored at $4^{0} \mathrm{C}$.

\section{Inocolum preparation}

Bacterial cells obtained from laboratory stock solution would be inoculated into $10 \mathrm{~mL}$ of $\mathrm{LB}$ broth. This would be incubated with shaking overnight at $37^{\circ} \mathrm{C}$. The inoculum density of the bacterial cells was done using UV spectrophotometer to determine its absorbance.

\section{Water quality determination}

Raw water collected from Sungai Gombak was characterized to determine the various parameters of water in order to bench mark against the raw water quality requirement as stated in the drinking water quality standard by the ministry of health Malaysia. The parameters determined were explained in the following sub sections.

\section{Heterotrophic plate count (HPC)}

This test would provide a general measure of microorganism activity in water. This was done by taking $1 \mathrm{~mL}$ of the sample into a petri dish and certain quantity of molten LB agar was poured. After the agar was solidified, the dish was incubated at $37^{\circ} \mathrm{C}$ for 24 hours. The colonies were counted to compute the concentration of the bacteria presented.

\section{Artificial neural network modelling}

Artificial neural networks have been identified to be useful for tasks that involved incomplete data sets and for highly complex and illdefined problems, where humans usually made decisions based on an intuitional basis. Their abilities to derive meaning from complicated or imprecise data allowing them to extract patterns and detect trends that were too complicated to be noticed by humans or conventional computer techniques, especially for non-linear relationship (Hanbay et al., 2008).

\section{Disinfection kinetic models}

The disinfection kinetic models were carried out and the residual colonies were estimated at given time intervals at constant agitation and optimum dosage. The experimental data collected was used to fit the different disinfection inactivation models such as the Chicks, Watson, Collin-Selleck and Homs models. The result with the highest coefficient of determination was chosen as the best fitted. Also, the statistical analysis for the inactivation kinetics of the seed extract was examined by means of non-linear multivariate regression analysis with respect to the bacterial strains. The log survival ratio was chosen as the dependent variable while dosage and time taken were chosen as the predictors. The student t-test was used to assess the significance level of the regression coefficient (Azzellino et al., 2011).

\section{Statistical analysis}

All the results obtained from this study except where otherwise stated, were carried out in triplicates hence, their mean values were determined with Microsoft Excel or SPSS software version 20. The significance levels of the generated data in this study were also determined with SPSS (v.20).

\section{Jar test}

The jar test was used to determine the optimum Moringa oleifera seed extract dosage required for the coagulation and flocculation process. The result obtained was used in the bench scale treatment of water. The operating variables used for the jar test were 2 minutes for the rapid mixing at $110 \mathrm{rpm}$ agitation rate, 25 minutes for the slow mixing at $30 \mathrm{rpm}$ agitation rate and 60 minutes for the sedimentation time.

\section{Application to surface river water}

A bench scale model consisting of five treatment units was developed in order to validate the optimum disinfectant dosage using a typical river collected from Sungai Gombak. The unit operations used in the model were coagulation, flocculation, sedimentation, sand filtration and disinfection processes. The bench scale treatment unit was operated in a continuous mode and the raw water collected from Sungai Gombak was kept in an overhead tank in order to filter out sediment large particles as the supernatant was passed to the coagulation and flocculation units using Moringa oleifera seed extracts as the coagulant. The supernatant from the sedimentation unit was discharged into the sand filtration unit to further remove suspended solids. The resulting solution from the filtration unit was passed to the disinfection tank where Moringa oleifera seed extract was used to disinfect the water based on the optimum values obtained.

\section{RESULTS AND FINDINGS}

The best parameters for training in the model were one hidden layer and ten number of hidden neurons. The training functions used were the Leven-Marquardt (TRAINLM) with gradient descent momentum weight/bias (LEARNGDM) learning function, transfer functions of Tan-sigmoid (TANSIG) in the hidden layer and pure linear (PURELIN) transfer function in the output layer. The data obtained during the disinfection conditions for E.coli bacterial strain was supplied into the ANN model. It was trained to achieve the highest multiple correlation coefficients R. The results were summarized in Fig. 1.

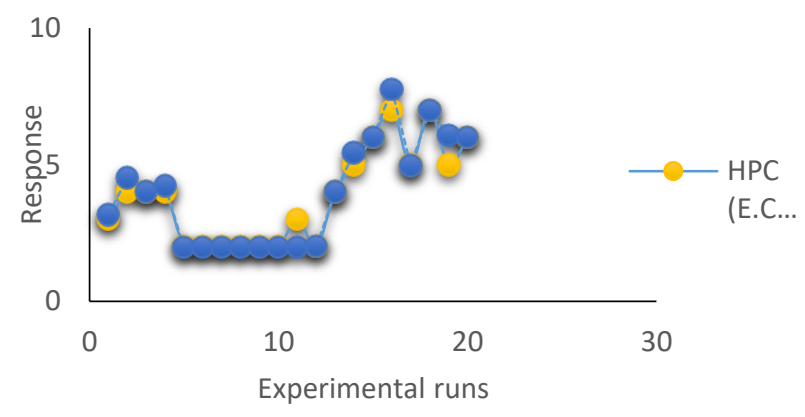

Fig. 1 Results for predicted output for E.coli.

\section{Training of ANN models for E.coli}

The data obtained during the optimization of the process disinfection conditions for E.coli bacterial strains was supplied into the ANN model. It was trained to achieve the highest multiple correlation coefficients R. The output from ANN model obtained was also compared with the experimental data and the predicted output from RSM. At the model level, the correlation measurements for the estimation of the regression equation were the multiple coefficients $R$ and the determination coefficient $\mathrm{R}^{2}$. The correlation between the experimental and the predicted values was better when $\mathrm{R}$ was closer to 1. The results as shown in Fig. 1 showed that the value of $R$ and $R^{2}$ were 0.99428 and 0.9886 , respectively for the optimum disinfection process. These values showed a high degree of correlation between the predicted outputs and the experimental outputs. The value of $\mathrm{R}^{2}$ showed that about $98.86 \%$ of the process conditions were significantly affected the disinfection process in a positive way. The value of $\mathrm{R}^{2}$ was also indicated for the measurement fit of the model and it revealed that only about $1.14 \%$ of the total variations could not be explained by the model. 

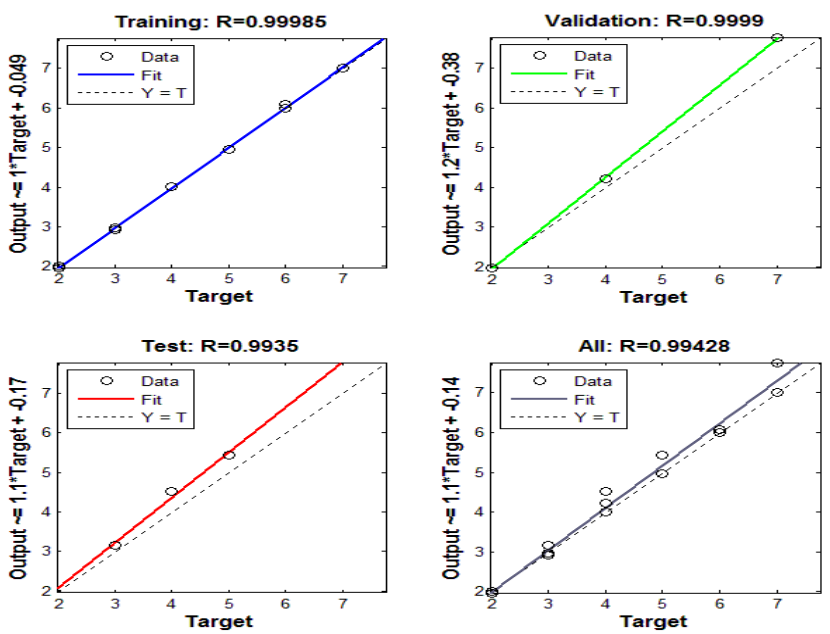

Fig. 2 Regression plot with the result of R value for HPC of E.coli cells.

The network performance was analysed in terms of its meansquared error (MSE) which was the square of the average difference between actual output and desirable output. The lower the MSE value towards zero, the better the performance. Network training was stopped automatically if the validation error was continued to increase. The best performance was seen at 0.2095 at epoch 1 because the training error was continued to decrease while the test errors and validation errors were continued to increase and hence, the network was automatically stopped. The result was reasonable because the mean square error is was small, the test set error and the validation set error have similar characteristics and no significant over-fitting was occurred. The MSE of the ANN model was 0.2044 which indicated that the error of the model was small and hence, giving a better and more accurate prediction of the output during the disinfection process.

\section{Sensitivity analysis}

Sensitivity analysis was done to determine the significant effects of each parameters or inputs on the process conditions of disinfection and since ANN became a black box model, it did not give such insights of the system directly. But there were numerous methods available which gave the sensitivity analysis of the system using inherent nature of ANN. Fig. 3 shows the ANN sensitivity analysis of the system using 'per- turb method'. Each series in the graph was represented for the rate of change of response with change in the given input variable. Higher the slope and the range of change in the response, greater the influence of the variable. It also revealed on how the model would respond to changes in the values of the parameters (Desai et al., 2008). The closer $r$ value to 1 and the lesser the p-value $(<0.05)$ were indicated for the more significant effects of inputs on the output. The results were plotted in form of a graph as shown in Fig. 3 for E.coli. The sensitivity analysis of the E.coli bacterial strain showed that dosage was highly significant, followed by contact time. However, agitation gave a negative $r$-value which indicated for a negative relationship and hence, effect of agitation was insignificant in the model.

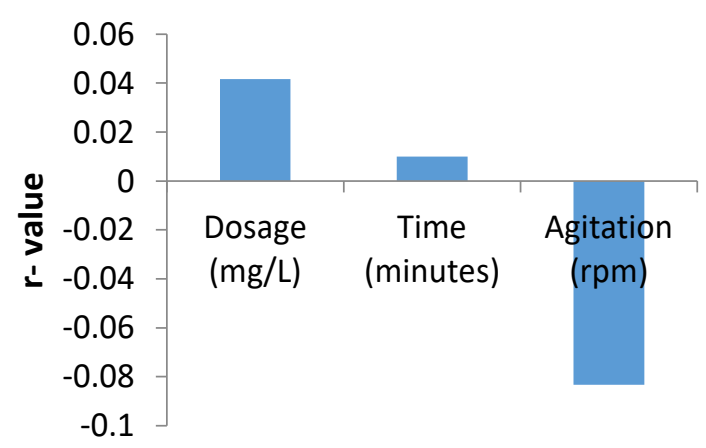

Fig. 3 Sensitivity analysis plot of HPC for E.coli

\section{Optimization of process parameters using ANN model}

The trained ANN models for the HPC of E.coli were used to find the optimum inputs. The trained ANN models for data from HPC for E.coli that gave $\mathrm{R}^{2}$ value equal to 0.9998 was used to find the optimum inputs of the disinfection variable that would give minimum reduction in the bacterial cells. Three sets of optimal inputs range were obtained from the ANN model. The results for the optimum range of the parameters were shown in Table 2 and the surface plots of the optimum ranges were shown in Fig. 4.

Table 2 Optimum inputs for disinfection process of E.coli.

\begin{tabular}{ccccc}
\hline No & Experiment & Dosage $(\mathbf{m g} / \mathbf{L})$ & Time $(\mathbf{m i n})$ & Agitation $(\mathbf{r p m})$ \\
\hline 1 & EANN1 & 123.6 & 68 & 140 \\
2 & EANN2 & 126 & 69 & 135 \\
3 & EANN3 & 124 & 65 & 110 \\
\hline
\end{tabular}

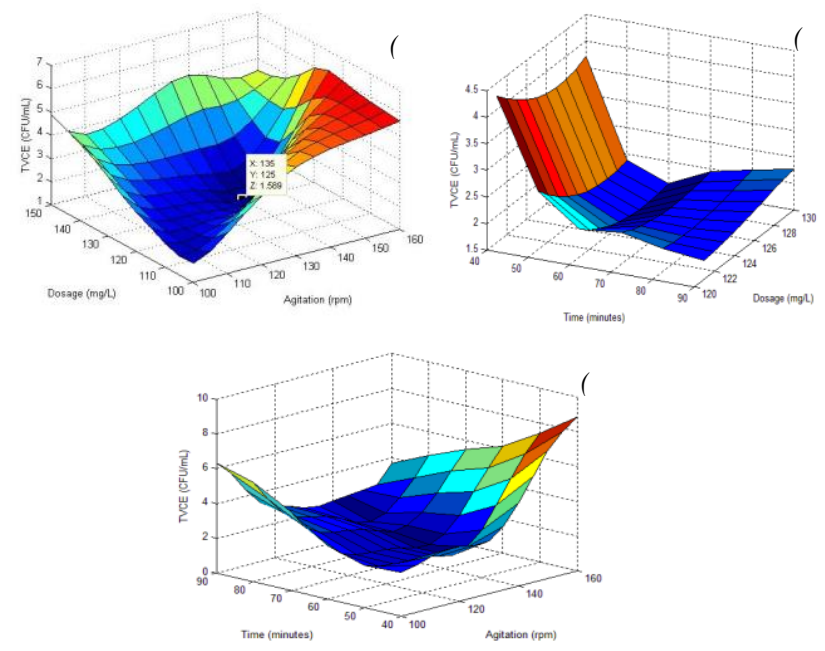

Fig. 4 Output surface plots for (a) dosage and agitation at constant time (b) contact time and dosage at constant agitation (c) Agitation and time at constant dosage of E.coli cells.

The surface plot of input variables dosage and agitation at constant time of 60 minutes on the disinfection process conditions which indicated a non-linear interaction. As the dosage was increased and agitation was increased, the HPC for E.coli was also increased. However, a local minimum region was observed at dosage range of $120 \mathrm{mg} / \mathrm{L}-130 \mathrm{mg} / \mathrm{L}$ and agitation range of $130 \mathrm{rpm}-140 \mathrm{rpm}$. In Fig. 4(b), increasing the time would lead to decrease in HPC for E.coli while little change was observed when increasing the dosage. Similar trend was observed in Fig. 4(c), whereby increasing the input variables (both agitation and time) would increase the heterotrophic plate count for E.coli. The results from the surface plots Fig. 4(a-c) showed that the optimum range of Moringa oleifera dosage was about from 120 to 130 $\mathrm{mg} / \mathrm{L}, 60$ to 70 minutes for the contact time range and from 100 to 140 $\mathrm{rpm}$ for the agitation range.

\section{Validation of ANN models}

The validation of the ANN models for E.coli cells was summarized in Table 3. Good agreement between the experimental and predicted results verified the models validity while the optimal conditions achieved for E.coli cells were $125 \mathrm{mg} / \mathrm{L}, 65$ minutes and $110 \mathrm{rpm}$ agitation.

Table 3 Validation of ANN model of E.coli.

\begin{tabular}{lllllll}
\hline No & Experiment & $\begin{array}{l}\text { Dosage } \\
\text { (mg/L) }\end{array}$ & $\begin{array}{l}\text { Time } \\
\text { (minutes }\end{array}$ & $\begin{array}{l}\text { Agita } \\
\text { tion } \\
\text { (rpm) }\end{array}$ & $\begin{array}{l}\text { Experim } \\
\text { ental } \\
\text { output }\end{array}$ & $\begin{array}{l}\text { Predict } \\
\text { ed } \\
\text { output }\end{array}$ \\
\hline 1 & ANNI & 123.6 & 68 & 140 & 2 & 1.12 \\
2 & ANN2 & 126 & 69 & 140 & 2 & 1.22 \\
3 & ANN3 & 125 & 65 & 110 & 1 & 1.33 \\
\hline
\end{tabular}




\section{Inactivation kinetics of synthetic water containing E.coli}

The plot of log survival of the E.coli bacterial colonies against time showed a deceleration of the process called "tail" as shown in Fig. 5. It was hypothesized that tailing was only occurred when a (small) subpopulation of a microbial population was not effectively inactivated as a result of clumping during the disinfection exposure (Pennell et al., 2008). Another possible explanation might be as a result of decrease in the germicidal properties of defatted Moringa oleifera salt extract with time. From the plot, it was observed that the bacteria population was gradually to build up over a period of time which might be as a result of resistance among the microorganisms. This was also observed in a study conducted by (Nwaiwu \& Lingmu, 2011) where tailing was also observed using Moringa oleifera seed extract for the treatment of both medium and low turbid water. This observation in terms of the efficiency if the seed extract showed that over a period of time, the disinfectant ability could be decreased which enhancing the resistance of the microorganisms against it.

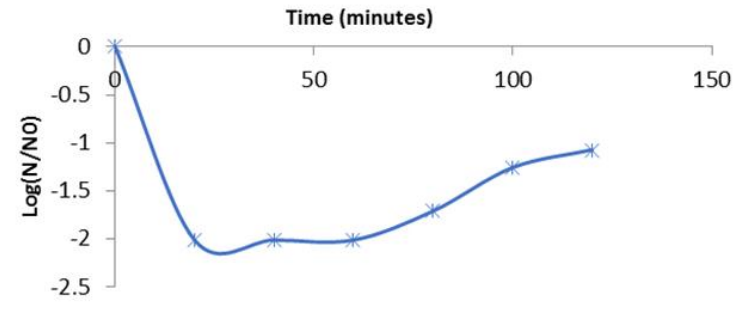

Fig. 5 Plot of log survival of E.coli bacterial cells against time.

Hence, to determine the disinfection kinetics of Moringa oleifera seed extract, the goodness of fit by comparing the coefficient of determination $\left(\mathrm{R}^{2}\right)$ for Chick-Watson model, Hom's model and Selleck model were carried out using multiple linear regressions in IBM SPSS 20 statistical software. The least square methods were used and the significance level of the regression coefficient was assessed by means of the t-test. The summary of the coefficient of determination of the results was shown in Table 4.

Table 4 Coefficient determination for different disinfection kinetic models.

\begin{tabular}{lcc}
\hline Disinfection kinetic model & $\mathbf{R}^{2}$ & Adjusted $\mathbf{R}^{\mathbf{2}}$ \\
\hline Chick-Watson & 0.309 & 0.171 \\
Selleck & 0593 & 0.511 \\
Hom & 0.711 & 0.689 \\
\hline
\end{tabular}

From the experimental data, the Hom empirical model was best described the data and since $m$ was less than 1, tailing was observed as confirmed from Fig. 4. The Homs model was a regression of disinfection performance against the two important factors, time and disinfectant concentration. Both the contact time and concentration were statistically significant as indicated in Table 4 although, time was still the most important parameter $(\mathrm{P}<0.000)$. The coefficient of determination $\mathrm{R}^{2}$ obtained was 0.7111 and adjusted $\mathrm{R}^{2}$ obtained was 0.689 .

\section{Coagulation-flocculation-sedimentation process}

Report from previous research showed that defatted crude Moringa oleifera seed extract could treat low turbidity. The dosage applied was varied from $5 \mathrm{mg} / \mathrm{L}$ to $40 \mathrm{mg} / \mathrm{L}$ and the results were in average value of 15 experimental data. The parameters monitored were turbidity and colour, which indicating the physical properties of water, while the heterotrophic plate count (HPC) and total coliform (TC) were indicated for the microbiological quality of the water sources. The extract was prepared from fresh every time for the water treatment. The effects of Moringa oleifera seed extract on the water quality showed the reduction in turbidity as the seed dose was increased but the optimum dosage obtained was between $10 \mathrm{mg} / \mathrm{L}$ to $20 \mathrm{mg} / \mathrm{L}$ with the final turbidity in range of $2.49 \mathrm{NTU}$ to $2.58 \mathrm{~N} \mathrm{TU}$. The seed extracts contained significant amount of low molecular weight (water soluble) proteins that possessed positive charges when they were added to raw water and these positive charges could act as magnets to attract the predominantly negatively charged particles (such as clay, silk, bacteria and other toxic particles in water) (Santos et al., 2013). During the flocculation process, the proteins would bind with the negative charge to form flocs through the aggregation of particles which were presented in water. These flocs were removed by settling or filtration which could be responsible for the drastic reduction in bacterial concentration. Increasing the dosage beyond the optimum did not improve the removal of turbidity because large dose of the seed extract could cause restabilization of particles as a result of the little particles that left in the water which were insufficient to form interparticle bridges (Mwh, 2005). The result obtained was supported by earlier research by (Muyibi et al., 2004) whose optimum dosage obtained was $20 \mathrm{mg} / \mathrm{L}$. For the reduction of the colour, the optimum dosage range for colour reduction was around $10 \mathrm{mg} / \mathrm{L}$ to $20 \mathrm{mg} / \mathrm{L}$ which gave 10 units $\mathrm{PtCo}-11$ units PtCo for the water. It was observed that the TDS and electrical conductivity values were increased slightly by the increasing doses of the seed extract and hence, it could be concluded that the seed extract has insignificant effect on these two parameters. This result was in close agreement with earlier research conducted by (Arnoldsson et al., 2008). It could be concluded that Moringa oleifera seed extract has no significant effect on $\mathrm{pH}$ of water (Kumar et al., 2012), Thus, as a result, the pH of the water was not monitored. The reduction in the bacterial population as indicated from the TC and HPC showed that high reduction was achieved at the optimum dose range of $10 \mathrm{mg} / \mathrm{L}$ to $20 \mathrm{mg} / \mathrm{L}$. The total coliform was reduced from $2400 \mathrm{MPN} / 100 \mathrm{~mL}$ to $34 \mathrm{MPN} / 100 \mathrm{~mL}$ while the heterotrophic plate count was reduced from $6500 \mathrm{CFU} / \mathrm{mL}$ to $70 \mathrm{CFU} / \mathrm{mL}$. These results showed that the effectiveness of coagulation-flocculation process was drastically reducing these microbial populations. Based on the coagulation optimization of this research, the range of $10 \mathrm{mg} / \mathrm{L}$ to $20 \mathrm{mg} / \mathrm{L}$ of the defatted crude Moringa oleifera seed extract was able to reduce most of the water parameters. Hence, for the jar tests, the defatted crude seed extract was used as the coagulant while the range of the optimum dose was accounted for the fluctuating water quality which occurred as a result of the monsoon season at the time of the experiments.

\section{Disinfection process and removal efficiency}

After the treatment with the defatted seed extract during the coagulation process, the effluent collected was disinfected. It was important to note that this method has not been reported in any study in which the extract of the seed was used as a coagulant and disinfectant. It was advantageous because using the same material as disinfectant and coagulant could reduce cost; it was eco-friendly, readily available and non-toxic. The defatted seed extract extracted with $1 \mathrm{M}$ salt solution extraction was used as the disinfectant. Using the optimum ANN model generated, the required disinfection dose was calculated based on the number of bacterial colonies left in the effluent from the coagulation process. The dosage applied during the disinfection process was varied throughout the experiments due to the fluctuating quantity of the bacterial colonies left in the water. The experimental data summarized in Table 5 was mean values of 15 data.

Table 5 Mean values of water parameters after disinfection.

\begin{tabular}{llll}
\hline Parameters & Range value & Mean value & Std Dev \\
\hline Turbidity (NTU) & $2.8-6.8$ & 5.06 & 1.33 \\
Colour (PtCo) & $7-14$ & 11 & 1.5 \\
HPC (CFU/mL) & $2-20$ & 9.8 & 5.7 \\
TC (MPN/100mL) & $0-2$ & 1.2 & 0.72 \\
TDS (mg/L) & $33-46.4$ & 39.5 & 5.21 \\
\hline
\end{tabular}

The results summarized in Table 5 showed the water parameters and it was observed that the turbidity was 5.06NTU, colour was reduced to 11 units PtCo, HPC and TC were reduced to $9.8 \mathrm{CFU} / \mathrm{mL}$ and $1 \mathrm{MPN} / 100 \mathrm{~mL}$, respectively and the total dissolved solid was $39.5 \mathrm{mg} / \mathrm{L}$. The removal efficiency carried out showed the extent in which the parameters monitored were reduced. The parameters (colour, COD, turbidity, HPC, TC) were measured after the disinfection process. The results revealed that to a greater level, most of the parameters monitored were reduced. For the COD, reduction about $50 \%$ was achieved while $66 \%$ and $50 \%$ reduction were achieved for 
turbidity and colour, respectively. This average efficiency showed that moringa seed extract has low coagulation activity for low turbid water and this observation has been noted by earlier research (Madrona et al., 2012). For HPC and TC, 98.67 and 98.58 percentage reduction were achieved, respectively. This showed that defatted Moringa seed extract used in the coagulation process could drastically reduce the bacterial count as well as the coliform count. For both the TDS and conductivity, there was slight increase in their values and hence, the percentage reduction was not significant. However, during the disinfection process, percentage reduction of turbidity and colour were reduced to $15.67 \%$ and $30 \%$, respectively. This was because dosing more extract could cause restabilization due to insufficient particles left in the water although the final turbidity was still less than the original sample (Nkurunziza et al., 2009). For the HPC and TC, microbial population was reduced during the disinfection process and the removal efficiencies were $99.91 \%$ and $99.92 \%$, respectively. This clearly showed the disinfection ability of the seed extract. Other researchers have pointed that an antibacterial agent was presented in the seed which gave it ability to inhibit microorganisms (Ferreira et al., 2011).

\section{CONCLUSION}

The use of ANN could approximate functions to any desired degree of accuracy and it could be used for complex non-linear relationship. The interactions in ANN were shown both qualitatively and quantitatively by examining the prediction values of supplied inputs and targets data. ANN was not used for experimental design but it could be used to analyse the results obtained from the experiment to achieve good and reliable results with low error output. The findings from the application of the seed extract to river water showed that the removal efficiency for the seed extract was over $99.98 \%$ reduction of heterotrophic bacteria after the disinfection process. Hence, the findings of this study showed that defatted Moringa oleifera seed extract using the salt extraction method could be used as a disinfectant. This extract was recommended to be used in small communities and in emergency situations.

\section{ACKNOWLEDGEMENT}

The authors were grateful to the Ministry of Higher Education (MOHE) Malaysia, for financing the research project under the research initiative grant (RIGS16-075-0239) and to Department of Biotechnology Engineering, International Islamic University Malaysia for their support.

\section{REFERENCES}

Alvarez, E. G. 2006. Artificial Neural Networks. Retrieved from http://edugi.unimuenster.de/eduGI.LA2/downloads/02/ArtificialNeuralNet works240506.pdf

Arnoldsson, E., Bergman, M., Matsinhe, N., Persson, K. M. 2008. Assessment of drinking water treatment using moringa oleifera natural coagulant. Vatten, 64, 137-150.

Azzellino, A., Antonelli, M., Canziani, R., Malpei, F., Marinetti, M., Nurizzo, C. 2011. Multivariate modelling of disinfection kinetics: A comparison among three different disinfectants. Desalination and Water Treatment, 29, $1-3,128-139$.
Bichi, M. H., Agunwamba, J. C., Muyibi, S. A. 2012. Optimization of operating conditions for the application of moringa oleifera (zogale) seeds extract in water disinfection using response surface methodology. African Journal of Biotechnology, 11, 92, 15875-15887.

Demuth, H., Beale, M., Hagan, M. 2008. Neural network toolbox 5 user 's guide. Retrieved from file:///C:/Users/User/Downloads/MATLAB__Neural_Network_Toolbox_5_-_Users_Guide_2007.pdf

Desai, K. M., Survase, S. A., Saudagar, P. S., Lele, S. S., Singhal, R. S. 2008. Comparison of artificial neural network (ANN) and response surface methodology (RSM) in fermentation media optimization: Case study of fermentative production of scleroglucan. Biochemical Engineering Journal, 41, 3, 266-273.

Ferreira, R. S., Napoleão, T. H., Santos, A. F. S., Sá, R. A., Carneiro-da-Cunha, M. G., Morais, M. M. C., et al. 2011. Coagulant and antibacterial activities of the water-soluble seed lectin from moringa oleifera. Letters in Applied Microbiology, 53, 2, 186-192.

Haykin, S. 1999. Neural Networks: A Comprehensive Foundation. McMaster University, Ontario Canada: Pearson.

Kumar, V. K., Rubha, M. N., Manivasagan, M., Babu, R., Balaji, P. 2012. Moringa oleifera - the nature' $\mathrm{s}$ gift abstract. Universal Journal of Environmental Research and Technology, 2, 4, 203-209.

Lippmann, R. P. 1987. An introduction to computing with neural nets. IEEE ASSP Magazine, 3, 4, 4-22.

Madrona, G. S., Branco, I. G., Seolin, V. J., Alves Filho, B. D. A., FagundesKlen, M. R., Bergamasco, R. 2012. Evaluation of extracts of moringa oleifera lam seeds obtained with nacl and their effects on water treatment. Acta Scientiarum. Technology, 34, 3, 289-293.

Mahajan, R. K., Walia, T. P., Lark, B. S., Sumanjit. 2006. Analysis of physical and chemical parameters of bottled drinking water. International Journal of Environmental Research and Public Health, 16, 2, 89-98.

Muyibi, S. A., Birima, A. H. M., Mohammed, T. A., Noor, M. J. M. M. 2004 Conventional treatment of surface water using moringa oleifera seeds extract as a primary coagulant. IIUM Engineering Journal, 5, 1, 25-35.

Mwh. 2005. Water treatment: Principles and Design (Second Edi ed.). United States of America: John Wiley \& Sons.

Nkurunziza, T., Nduwayezu, J. B., Banadda, E. N., Nhapi, I. 2009. The effect of turbidity levels and moringa oleifera concentration on the effectiveness of coagulation in water treatment. Water Science and Technology, 59, 8, 15511558 .

Nwaiwu, N. E., Lingmu, B. 2011. Studies on the effect of settling time on coliform reduction using moringa oleifera seed powder. Journal of Applied Sciences in Environmental Sanitation, 6, 3, 279-286.

Pal, M. P., Vaidya, B. K., Desai, K. M., Joshi, R. M., Nene, S. N., Kulkarni, B. D. 2009. Media optimization for biosurfactant production by rhodococcus erythropolis mtcc 2794: Artificial intelligence versus a statistical approach. Journal of Industrial Microbiology \& Biotechnology, 36, 747-756.

Pennell, K. G., Aronson, A. I., Blatchley, E. R. 2008. Phenotypic persistence and external shielding ultraviolet radiation inactivation kinetic model. Journal of applied microbiology, 104, 4, 1192-1202.

Ricca, R. N., Jami, M. S., Alam, M. Z. 2012. The potential of artificial neural network (ANN) in optimizing media constituents of citric acid production by solid state bioconversion. International Food Research Journal, 19, 2 , 491-497.

Santos, A. F. S., Paiva, P. M. G., Teixeira, J. A. C., Brito, A. G., Coelho, L. C. B. B., Nogueira, R. 2013. Coagulant properties of moringa oleifera protein preparations: Application to humic acid removal. Environmental technology, 33, 3, 69-75.

Wu, W., Dandy, G. C., Maier, H. R. 2011, 2011. Application of artificial neural networks to forecasting water quality in a chloraminated water distribution system. 19th International Congress on Modelling and Simulation. 12-16 December. Perth, 1112-1118. 